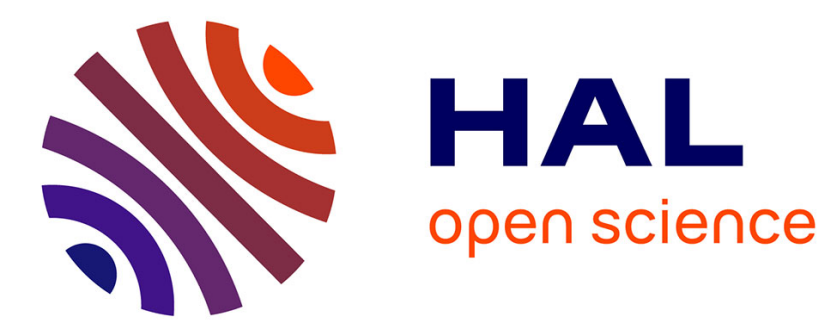

\title{
Estimating the infection rate of a SIR epidemic model via differential elimination
}

\author{
Rosane Ushirobira, Denis Efimov, Pierre-Alexandre Bliman
}

\section{To cite this version:}

Rosane Ushirobira, Denis Efimov, Pierre-Alexandre Bliman. Estimating the infection rate of a SIR epidemic model via differential elimination. ECC 2019 - 18th European Control Conference, Jun 2019, Naples, Italy. hal-02418553

\section{HAL Id: hal-02418553 \\ https://hal.inria.fr/hal-02418553}

Submitted on 18 Dec 2019

HAL is a multi-disciplinary open access archive for the deposit and dissemination of scientific research documents, whether they are published or not. The documents may come from teaching and research institutions in France or abroad, or from public or private research centers.
L'archive ouverte pluridisciplinaire HAL, est destinée au dépôt et à la diffusion de documents scientifiques de niveau recherche, publiés ou non, émanant des établissements d'enseignement et de recherche français ou étrangers, des laboratoires publics ou privés. 


\title{
Estimating the infection rate of a SIR epidemic model via differential elimination
}

\author{
Rosane Ushirobira $^{1}$, Denis Efimov ${ }^{1}$, Pierre-Alexandre Bliman ${ }^{2}$
}

\begin{abstract}
A SIR epidemic model is one of the most wellknown mathematical models that helps to understand the dissemination of an infectious illness. It is a three-compartment model composed by individuals that are susceptible, infective and recovered with respect to the disease. In this work, the infection rate is estimated for a particular SIR epidemic model by using as the output measurement the incidence rate, which is a nonlinear function of the state variables. The aim is then to eliminate variables in the given system for which there are no measurements, such as the proportion of each type of individuals (susceptible, infective and recovered). The method applied here is based on differential elimination concepts from differential algebra, more precisely the Rosenfeld-Gröbner algorithm is employed. Once the input-output (IO) equation is determined, the derivatives of the signal are estimated by a homogeneous finite-time differentiator and a gradient descent method can be applied to solve the IO equation for the infection rate.
\end{abstract}

\section{INTRODUCTION}

In 1932, J. Ritt [17] founded the principles of differential algebra, a theory that allows the study of differential equations, ordinary or partial, within an algebraic framework. An important issue among the several developments of differential algebra is represented by the theory of differential elimination. This theory consists essentially in rewriting a system of differential equations into an equivalent one, taking into account a certain ordering of its variables and derivatives. Basically, the given ordering indicates what must be eliminated, allowing a certain control of the elimination procedure. The theory of differential elimination appeared naturally from early stages of differential algebra. In automatic control theory, an example of an application of differential elimination can be easily seen as a manner of obtaining a system of equations in the parameters to be estimated given in terms of measured quantities and their derivatives, by then eliminating state variables.

Among others, a quite remarkable algorithm for elimination is based on the Rosenfeld-Gröbner method. This algorithm was introduced by F. Boulier [4] and more complete details can be found for instance in [6]. Boulier used Gröbner bases to design the Rosenfeld-Gröbner algorithm and it was

\footnotetext{
${ }^{1}$ Rosane Ushirobira and Denis Efimov are with Inria, Univ. Lille, CNRS, UMR 9189 - CRIStAL, F-59000 Lille, France Rosane. Ushirobira, Denis.Efimoveinria.fr

${ }^{2}$ Pierre-Alexandre Bliman is with Inria, Sorbonne Université, Université Paris-Diderot SPC, CNRS, Laboratoire Jacques-Louis Lions, Paris, France Pierre-Alexandre.Blimaneinria.fr
}

how initially this algorithm was implemented in Maple. Numerous applications followed from this important algorithm, for instance in [5] the author describes differential elimination applied to mathematical problems, notably arising from biological examples, such as the parameter estimation in two-compartment problems or the model reduction for the circadian clock of the green alga Ostreococcus tauri.

Other useful differential algebra tools, especially Gröbner bases, were also used in control problems, such as for identifiability topics. Parameter identifiability and input estimation of nonlinear systems were studied for example in [16], [13]. Furthermore, a promising algebraic framework to parameter estimation relies on the concepts of integrodifferential algebras. The reader may find a study in this context in [7].

In the present paper, an algebraic approach is applied to analyze a biological mathematical model, a SIR model, which represents the spreading of an infectious disease. This three-compartment model is one of the most popular models to approximate the development of the illness. The estimation of the parameter describing the infection rate in a SIR model provides knowledge of the population and of the epidemic. The available measured output is the incidence rate, that is the number of new infective individuals per time unit, which is a nonlinear function of the state variables. Few works were devoted to this problem of infection rate estimation, such as in [2] for the time-varying case. In addition, interval observers were applied in [3] and more recently, the authors have designed a class of nonlinear adaptive observers for this model [15]. See also [12] for the design of a class of interval observers for vector-borne diseases.

This paper is constituted by the following sections. In Section II, we describe the epidemic model and the parameter estimation problem to be studied. Basic definitions and properties of differential algebra are introduced in Section III. Using these concepts, in Section IV we apply the RosenfeldGröbner algorithm to compute an estimate for the infection rate. This method is tested numerically. Conclusions follow in Section V. An Appendix summarizes some results on homogeneous finite-time differentiator, borrowed from [14]. 


\section{Problem STATEMEnt}

Consider a basic SIR model with demography [11]:

$$
\begin{aligned}
\dot{S}(t) & =\mu-\beta S(t) I(t)-\mu S(t), \\
\dot{I}(t) & =\beta S(t) I(t)-(\gamma+\mu) I(t), \\
\dot{R}(t) & =\gamma I(t)-\mu R(t), \\
y(t) & =\beta S(t) I(t),
\end{aligned}
$$

where the functions $S, I, R: \mathbb{R}_{+} \rightarrow[0,1]$ represent proportions of susceptible, infected and recovered individuals, respectively; $0<\mu<\gamma<+\infty$ are known parameters, representing the mortality ${ }^{1}$ and recovery rates; $\beta \in \mathbb{R}_{+}$is a unknown parameter corresponding to the infection rate, and the measured output available is given by the function $y: \mathbb{R}_{+} \rightarrow \mathbb{R}_{+}$. The aim of this work is to estimate the value of the infection rate function $\beta$, based on continuous measurement of $y(t)$.

As mentioned previously, $S, I$ and $R$ are proportions of the constant total population, that is:

$$
S(t)+I(t)+R(t)=1 \quad \forall t \geq 0 .
$$

So since the total population is constant, we can then exclude the dynamics of $R$ in our computations. For this reason, omitting $R$ from (1), the simplified model becomes:

$$
\begin{aligned}
\dot{S}(t) & =\mu-y(t)-\mu S(t), \\
\dot{I}(t) & =y(t)-(\gamma+\mu) I(t), \\
y(t) & =\beta S(t) I(t) .
\end{aligned}
$$

The proportion of susceptibles and infectious are not directly accessible to measurement. So the idea is to eliminate these unknown variables from (2) to obtain input-output (IO) equations depending only on the parameters and on the known quantity $y$ and its derivatives. These are the equations that will provide formulas for the estimate of $\beta$.

For the first step, elimination theory methods from differential algebra will determine the input-output equations. In the next Section, differential algebra and related topics used here are recalled, before applying in Section IV these techniques to the problem under study.

\section{Differential ALgEBRA CONCEPTS}

Some definitions and structural properties of differential algebra are given in this part. More details can be found in Ritt's seminal book [17].

Let $\mathcal{R}$ be a commutative ring with unity. Recall that a derivation $D: \mathcal{R} \rightarrow \mathcal{R}$ is a map that satisfies $D(a b)=D(a) b+$ $a D(b)$ and $D(a+b)=D(a)+D(b), \forall a, b \in \mathcal{R}$. An element $c \in \mathcal{R}$ is a constant if $D(c)=0$.

\section{Definition 1:}

1) A differential ring $\mathcal{R}$ is a commutative ring with unity equipped with commuting derivations $D_{1}, \ldots, D_{n}$. We

\footnotetext{
${ }^{1}$ As it is common in this simple model (SIR), it is assumed that there is a natural host lifespan of $\frac{1}{\mu}$ years. So the rate of natural mortality is given by the disease-independent factor $\mu$ [11].
}

write it sometimes $\left(\mathcal{R} ; D_{1}, \ldots, D_{n}\right)$. A differential ring $\mathcal{R}$ equipped with a single derivation $D$ is an ordinary differential ring.

2) A differential ideal $I$ of a differential ring $\left(\mathcal{R} ; D_{1}, \ldots, D_{n}\right)$ is an idea ${ }^{2}$ satisfying $D_{i}(I) \subset I$, $\forall i=1, \ldots, n$.

Now, given a polynomial ring $\mathcal{R}=\mathbb{K}\left[u_{1}, \ldots, u_{n}\right]$ on the indeterminates $u_{1}, \ldots, u_{n}$ and coefficients in the field $\mathbb{K}$ $\left(\mathbb{K}=\mathbb{R}\right.$ or $\mathbb{C}$ ), consider $D_{1}, \ldots, D_{n}$ derivations of $\mathcal{R}$. A derivation operator of $\mathcal{R}$ is a monomial $D_{1}^{\delta_{1}} \ldots D_{n}^{\delta_{n}}$ with $\delta_{1}, \ldots, \delta_{n} \in \mathbb{N}$. A differential polynomial ring $\mathbb{K}\left\{u_{1}, \ldots, u_{n}\right\}$ on the differential indeterminates $u_{1}, \ldots, u_{n}$ is the (usual) commutative polynomial ring on $u_{1}, \ldots, u_{n}$ and $\Delta\left(u_{i}\right)$ where $\Delta$ is a derivation operator of $\mathcal{R}$ and $i=1, \ldots, n$. Denoting by $U$ the set of variables $u_{1}, \ldots, u_{n}$, then the differential polynomial ring $\mathbb{K}\left\{u_{1}, \ldots, u_{n}\right\}$ can be also written as $\mathbb{K}\{U\}$.

Example 1: An illustrative example can be found in Ritt's book [17] and it is given by the ordinary differential equation $h(u)=0$, with $h(u)=\dot{u}^{2}-4 u$ where $u$ is a variable depending on the time $t$. The differential polynomial $h$ belongs to the differential polynomial ring $\mathbb{Q}\{u\}$ equipped with the derivation ' (meaning derivation with respect to $t$ ). The solutions of this ODE are $u(t)=(t+K)^{2}$ ( $K$ constant) and $u(t)=0$. Denote by $\mathfrak{a}$ the differential ideal generated by $h$, so $\mathfrak{a}$ is generated by $h$ and all derivatives of $h$ with respect to $t$.

Taking the derivative of $h$, we obtain a new ordinary differential equation:

$$
2 \dot{u}(\ddot{u}-2)=0
$$

and we remark that the solutions $(t+K)^{2}$ and 0 are also solutions of this ODE. This is again valid for any higher order derivative of $h(u)=0$. Generally speaking, the solutions also hold for any finite linear combination of derivatives of $h$ with differential polynomials as coefficients. In other words, they hold for any element in the radical of $\mathfrak{a}$. 3

As mentioned in the Introduction, a very efficient algorithm in elimination theory is the Rosenfeld-Gröbner method. One of the main issues of this algorithm is to give a representation of the radical of a differential ideal generated by a set of ordinary differential polynomials. For that, it is necessary to have also a ranking to indicate which variables must be eliminated.

The notion of ranking is very important for algorithmic aspects of differential algebra. A ranking is a total ordering of the variables and their derivatives. So given a system $\mathcal{F}$ of differential polynomials and a ranking on the indeterminates and its derivatives, the Rosenfeld-Gröbner algorithm provides

\footnotetext{
${ }^{2}$ An ideal $I$ of a ring $R$ is a non-empty subset of $R$ that satisfies $I+I \subset I$ and $I R \subset I$

${ }^{3}$ The radical of an ideal $\mathfrak{c}$ in the commutative ring $\mathcal{R}$ is the set of all elements in $\mathcal{R}$ whose some power belongs to $\mathfrak{c}$.
} 
a decomposition of the radical of the differential ideal generated by $\mathcal{F}$. More precisely, the algorithm returns a finite family $\left\{C_{1}, \ldots, C_{r}\right\}$ of finite subsets of $\mathbb{K}\{U\} \backslash \mathbb{K}$, called differential chains. A differential ideal $\mathfrak{c}_{i}$ is associated to each $C_{i}$ by taking all elements in $\mathbb{K}\{U\}$ such that their pseudoremainder by $C_{i}$ is zero. ${ }^{4}$ With this notation, the radical $\mathfrak{r}$ of the differential ideal generated by $\mathcal{F}$ is decomposed as:

$$
\mathfrak{r}=\mathfrak{c}_{1} \cap \cdots \cap \mathfrak{c}_{r} .
$$

Example 2: In Ritt's example (see Example 1), applying the Rosenfeld-Gröbner algorithm to $\mathcal{F}=\{h(u)\}$ and the ranking $u<\dot{u}<\ddot{u}<\ldots$, it results in a decomposition of the radical $\mathfrak{r}$ of the differential ideal generated by $\mathcal{F}$ :

$$
\mathfrak{r}=\mathfrak{c}_{1} \cap \mathfrak{c}_{2}
$$

where $\mathfrak{c}_{1}=\{h(u)\}$ and $\mathfrak{c}_{2}=\{u\}$. The ideal $\mathfrak{c}_{1}$ corresponds to the solution $u(t)=(t+K)^{2}$ and $\mathfrak{c}_{2}$ to the trivial solution.

Elimination theory concepts in differential algebra thus provide a new manner to represent a system by eliminating state variables in a state-space representation and rewriting it into an input-output representation. Classical notions in control theory such as observability and identifiability can be tested by using these tools, see for instance [8], [9] and [13].

To further detail the elimination procedure to obtain IO equations, according to [18] let us consider a nonlinear dynamic system defined by time-dependent differential polynomials equations:

$$
\dot{x}(t)=f(x(t), u(t), \mathbf{p}), \quad y(t)=g(x(t), \mathbf{p}),
$$

with $x(t)=\left(x_{1}(t), \ldots, x_{n}(t)\right) \in \mathbb{R}^{n}$ is the state, $u(t) \in \mathbb{R}^{m}$ is the input and $y(t) \in \mathbb{R}^{r}$ is the measured output. The constant unknown parameter vector $\mathbf{p}$ belongs to $\mathbb{R}^{p}$ and $f$ and $g$ are polynomial functions of their arguments and may be rational in p. Consider $\mathcal{F}$ be the family of polynomials $\dot{x}(t)-f(x(t), u(t))$ and $y(t)-g(x(t))$. Choosing the following order relation

$u<\dot{u}<\ddot{u}<\cdots<y<\dot{y}<\ddot{y}<\cdots<x_{1}<x_{2}<\cdots<\dot{x_{1}}<\dot{x_{2}}<\ldots$,

a differential characteristic set is a family of differential polynomials of the form:

$$
\begin{aligned}
& A_{1}(u, y), \ldots, A_{r}(u, y), \\
& A_{r+1}\left(u, y, x_{1}\right), A_{r+2}\left(u, y, x_{1}, x_{2}\right), \ldots, A_{r+n}\left(u, y, x_{1}, \ldots, x_{n}\right) .
\end{aligned}
$$

This corresponds to the fact that the state variables were eliminated from the given system of equations and the first $r$ polynomial differential equations $A_{i}(u, y)=0, i=1, \ldots, r$ in $u$ and $y$ and its derivatives form the input-output relations of the system. The Rosenfeld-Gröbner algorithm is indeed an efficient tool to find these IO equations.

\footnotetext{
${ }^{4}$ For $f$ and $g$ polynomials, a pseudoremainder is the remainder of the pseudodivision of $f$ by $g$ (that is, the Euclidean division of $c^{\operatorname{deg}(f)-\operatorname{deg}(g)=1} f$ by $g$ where $c$ is the leading coefficient of $g$ ).
}

Example 3: Consider a linear two-compartment model without input of the type:

$$
\begin{aligned}
& \dot{x_{1}}=a x_{1}+b x_{2} \\
& \dot{x_{2}}=c x_{1}+d x_{2}
\end{aligned}
$$

where $x_{1}$ and $x_{2}$ are variables and $a, b, c$ and $d$ are parameters. Assume that only $x_{1}$ is observable. Apply differential elimination tools to obtain an IO equation in terms of $y=x_{1}$ and the result is:

$$
\ddot{y}-(a+d) \dot{y}+(a d-b c) y=0 .
$$

It is important to stress that singular solutions are included in the outcome of the Rosenfeld-Gröbner algorithm. A singular solution of an algebraic differential equation $p\left(t, x, \dot{x}, \ddot{x}, \ldots, x^{(n)}\right)=0$ of order $n$ is a solution that satisfies also the differential equation $\frac{\partial p}{\partial x^{(n)}}\left(t, x, \dot{x}, \ddot{x}, \ldots, x^{(n)}\right)=0$ (that is, the derivative of $p$ with respect to the differential indeterminate $x^{(n)}$ of highest order, called the separant of $p$ ). Hence, a singular solution satisfies a lower order equation that results from the elimination of $x^{(n)}$ in $p$ and $\frac{\partial p}{\partial x^{(n)}}$. The non-singular solutions are called general solutions.

Example 4: [10] Consider the algebraic differential equation given by

$$
p(t, x, \dot{x}, \ddot{x})=4 \dot{x}^{2} \ddot{x}^{2}-16 \dot{x}^{2} \ddot{x}+12 \dot{x}^{2}+16 x=0 .
$$

So $p$ is polynomial in $x$ and its derivatives. Singular solutions must satisfy $\frac{\partial p}{\partial x^{(n)}}(t, x, \dot{x}, \ddot{x})=8 \dot{x}^{2} \ddot{x}-16 \dot{x}^{2}=0$. Therefore, after the elimination of $\ddot{x}$ in $p$ and $\frac{\partial p}{\partial x^{(n)}}$, a singular solution has to fulfill

$$
\dot{x}^{2}-4 x=0 \text {. }
$$

There are two types of solutions for this equation $x(t)=$ $(t+a)^{2}$ and $x(t)=0$. These are the singular solutions of $p$.

\section{ESTIMATION}

The methods presented in the previous section are quite elaborated and do allow computations with more variables and complex interactions than in (1). To give a grasp on the soundness of differential-algebraic methods, our goal of this paper is to calculate IO equations for the system (2). That can be obtained by applying the Rosenfeld-Gröbner algorithm implemented in Maple.

In Maple, the DifferentialAlgebra package was used to define the differential ring with derivations in $t$ and the following variable order

$$
S>I>y .
$$

The Rosenfeld-Gröbner algorithm is implemented in Maple into the function RosenfeldGrobner. More precisely, for a given system of nonlinear differential equations (or inequations) sys and a differential ring $\mathrm{R}$ with a predefined ranking, the RosenfeldGroebner function returns a list of regular differential chains with respect to the ranking of $R$ 
(see Section III). So the first argument of this algorithm is a system of differential polynomial equations and the second one is a total ordering on the set of indeterminates.

Applying the Rosenberg-Gröbner algorithm, seven regular differential chains are obtained. That means that seven equations can be obtained by rewriting (2) according to the given order of variables. All solutions but one are singular, corresponding usually to equilibrium points. The nonzero singular solutions are the following:

$$
\begin{gathered}
\ddot{y}=\frac{\dot{y}^{2}-2 y^{3} \beta}{y} \\
y=\frac{\beta \mu-\mu^{2}}{\beta} \\
\dot{y}^{2}=-4 y^{3} \beta
\end{gathered}
$$

From (3), it follows

$$
\beta=\frac{\ddot{y} y-\dot{y}^{2}}{2 y^{3}}
$$

The expression (4) implies:

$$
\beta=\frac{\mu^{2}}{\mu-y}
$$

Finally, 5) provides:

$$
\beta=-\frac{\dot{y}^{2}}{4 y^{3}}
$$

The general solution resulting from the Rosenfeld-Gröbner algorithm is given by:

Theorem 1: The input-output equation of (1) is given by

$y(y-\mu) \ddot{y}^{2}-\left((2 y-\mu) \dot{y}^{2}-(y-2 \mu) \chi y \dot{y}-4 \beta y^{4}-\left(\chi^{2}-8 \mu \beta\right) y^{3}\right.$ $\left.+\left(\chi^{2}-4 \mu \beta\right) \mu y^{2}\right) \ddot{y}+\dot{y}^{4}-(\chi y-(2 \chi-\mu) \mu) \dot{y}^{3}-\left(4 \beta y^{2}+\right.$ $\left.\left(\chi^{2}-\chi \mu+(\mu-6 \beta) \mu\right) y-\left(\chi^{2}-3 \chi \mu+(\mu-3 \beta) \mu\right) \mu\right) y \dot{y}^{2}+$ $\left(2 \chi \beta y^{2}+\left(\chi^{2}-(\mu+2 \beta) \chi-2 \beta \mu\right) 2 \mu y+\left(-3 \chi^{2}+(\mu+\beta) 2 \chi+\right.\right.$ $\left.4 \beta \mu) \mu^{2}\right) y^{2} \dot{y}+4 \beta^{2} y^{6}+\left(\chi^{2}+4 \chi \mu-4(\mu+3 \beta) \mu\right) \beta y^{5}+$ $\left(\chi^{3}-(\mu+2 \beta) \chi^{2}-8 \beta \chi \mu+4(2 \mu+3 \beta) \mu \beta\right) \mu y^{4}+$$$
\left(-\chi^{3} \mu^{2}+(\mu+\beta) \chi^{2} \mu^{2}+4 \beta \chi \mu^{3}-4(\mu+\beta) \beta \mu^{3}\right) y^{3}=0
$$

where $\chi=\gamma+2 \mu$.

To estimate $\beta, 6$ is rewritten as a second-degree equation in $\beta$ :

$$
r \beta^{2}+f \beta+g=0
$$

with

$$
\begin{aligned}
r= & 4 y^{2}(y-\mu)^{2} \\
f= & 4 y(y-\mu) \ddot{y}-\left(4 y^{2}-6 \mu y+3 \mu^{2}\right) \frac{\dot{y}^{2}}{y-\mu} \\
& -2\left(\chi \mu-\chi y+2 \mu^{2}\right) y \dot{y}+\left(\chi^{2}+4 \chi \mu-4 \mu^{2}\right)(y-\mu) y^{2} \\
g= & \ddot{y}^{2}-\left((2 y-\mu) \dot{y}^{2}-\chi(y-2 \mu) y \dot{y}+\chi^{2} y^{2}(y-\mu)\right) \frac{\ddot{y}}{y(y-\mu)} \\
& +\frac{\dot{y}^{4}}{y(y-\mu)}-(y \chi-(2 \chi-\mu) \mu) \frac{\dot{y}^{3}}{y(y-\mu)} \\
& -\left(\left(\chi^{2}-\chi \mu+\mu^{2}\right) y-\left(\chi^{2}-3 \chi \mu+\mu^{2}\right) \mu\right) \frac{\dot{y}^{2}}{y-\mu} \\
& +(2(\chi-\mu) y-\mu(3 \chi+2 \mu)) \chi \mu \frac{y \dot{y}}{y-\mu}-\chi^{2} \mu(\mu-\chi) y^{2}
\end{aligned}
$$

A closed-form for the estimate of $\beta$ can then be obtained by solving the above second-degree equation. However, for applications such a solution may be problematic when $y=$ 0 or $y=\mu$. In that case, the nonlinear expression doesn't approach the linear expression. In order to avoid poles in the desired solution, a gradient descent method can be used in the following way. Consider the function:

$$
F(v)=r v^{2}+f v+g
$$

Define $G(v)=\frac{1}{2}\|F(v)\|_{2}^{2}$. Zeroing $F$ is equivalent to minimizing $G$. The gradient of $G$ being $F(v) F^{\prime}(v)$ provides the zeros of this latter function.

An important step in this estimation problem is also its numerical differentiation aspect since the first and second derivatives of $y$ appear in the expressions for $f$ and $g$ in (7). Many methods may be applied to estimate these derivatives, mostly within a statistics context. Among nonasymptotic techniques for numerical differentiation, algebraic methods appeared in the last twenty years, strongly based on differential algebra concepts. These methods were broadly applied to practical problems as in latency compensation for human-machine interaction or to detect marine bivalves spawning [19], [1]. Another very efficient non-asymptotic method is based on homogeneity concepts [14] whose effectiveness was proven through real-life applications as well. An important feature of these methods is their robustness to noise measurements. We adopt this later to estimate the derivatives in the illustration below, a brief description is given in the Appendix.

To illustrate the estimation method, we used the parameter values:

$$
\mu=\frac{1}{70} \text { year }^{-1} \text { and } \gamma=\frac{365}{7} \text { year }^{-1} \text {. }
$$

After applying the homogeneous finite-time differentiator described in the Appendix, Fig. 1 shows the error estimation for the reconstructed signal $y(t)$ from the noiseless simulated signal.

The whole procedure just discussed, highlights all benefits of symbolic-numerical methods. 


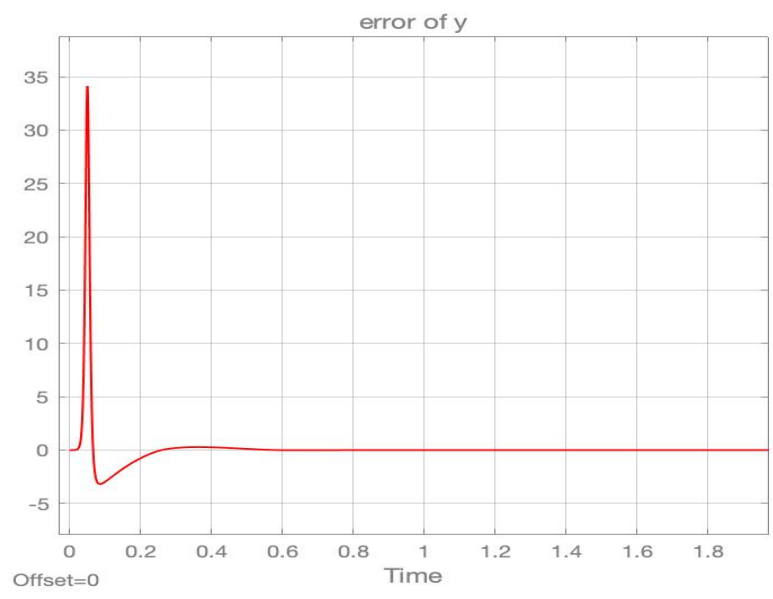

Fig. 1. The error of estimation for $y$

\section{CONCLUSION}

A parameter estimation problem has been considered in this paper for a SIR model (1). More precisely, a method for estimating the infection rate $\beta$ was proposed, based on the measured signal $y(t)=\beta S(t) I(t)$. An algebraic approach was applied to determine an input-output equation from the given nonlinear system, based on differential algebra theory, more precisely by applying the Rosenfeld-Gröbner algorithm, as a remarkable illustration of this theory potential. Using the resulting closed formula for $\beta$, a homogeneous numerical differentiator was applied to estimate the derivatives of $y(t)$ appearing in the formula. A part of future research includes the adaptation of more highly evolved numerical solutions to the quadratic equation that provides the estimate of the infection rate, taking into account that the system has no persistent excitation.

\section{ApPendiX: Homogeneous Finite-TIME DIFFERENTIATOR}

Consider a smooth signal $y(t)$. The goal of the homogeneous finite-time differentiator is to provide estimates for all derivatives up to order $n: \dot{y}(t), \ldots, y^{(n-1)}(t)$. Set $y^{(n)}(t)=$ $\theta(t)$ and $z=\left(\begin{array}{llll}y & \dot{y} & \cdots & y^{(n-1)}\end{array}\right)^{T}$. Therefore

$$
\dot{z}=A z+\Theta(t), \quad y=C z,
$$

with $A=\left(\begin{array}{ccccc}0 & 1 & 0 & 0 & 0 \\ 0 & 0 & 1 & 0 & 0 \\ \vdots & \vdots & \vdots & \ddots & \vdots \\ 0 & 0 & 0 & 0 & 1 \\ 0 & 0 & 0 & 0 & 0\end{array}\right), C=\left(\begin{array}{llll}1 & 0 & \cdots & 0\end{array}\right)$ and $\Theta(t)=\left(\begin{array}{llll}0 & \cdots & 0 & \theta(t)\end{array}\right)^{T} \in \mathbb{R}^{n}$. The homogeneous finitetime differentiator is proposed in [14]:

$$
\begin{aligned}
\dot{\widehat{z}}_{1} & =\widehat{z}_{2}-k_{1}\left\lceil\widehat{z}_{1}-y\right\rfloor^{\alpha}, \\
\dot{\widehat{z}}_{i} & =\widehat{z}_{i+1}-k_{i}\left\lceil\widehat{z}_{1}-y\right\rfloor^{i \alpha-(i-1)}, \quad i=2, \ldots, n-1, \\
\dot{\widehat{z}}_{n} & =-k_{n}\left\lceil\widehat{z}_{1}-y\right\rfloor^{n \alpha-(n-1)}
\end{aligned}
$$

where $\lceil x\rfloor^{\rho}:=|x|^{\rho} \operatorname{sign}(x)$ for all $x \in \mathbb{R}$ and $\rho>0, \widehat{z} \in \mathbb{R}^{n}$ is the estimate of $z$, the coefficients $k_{1}, \ldots, k_{n}$ define a Hurwitz polynomial and a choice $\alpha \in\left(0, n^{-1}\right)$ ensures the system homogeneity, for the weights $r_{i}=1-(i-1) \alpha$ for $i=1, \ldots, n$. Then, after some finite transient we obtain $\widehat{y}^{(j-1)}(t)=\widehat{z}_{j}(t)$, $j=1, \ldots, n$. The differentiation error dynamics is of the form:

$$
\begin{aligned}
\dot{e}_{1} & =e_{2}-k_{1}\left\lceil e_{1}\right\rfloor^{\alpha}, \\
\dot{e}_{i} & =e_{i+1}-k_{i}\left\lceil e_{1}\right\rfloor^{i \alpha-(i-1)}, \quad i=2, \ldots, n-1, \\
\dot{e}_{n} & =\theta(t)-k_{n}\left\lceil e_{1}\right\rfloor^{n \alpha-(n-1)} .
\end{aligned}
$$

The convergence of the error to zero is not possible without having some knowledge about the signal $\theta(t)$. However, by assuming that $y(t)$ is locally polynomial and that on a small time interval, $\theta(t)=0$, this problem can be bypassed and all time derivatives can then be estimated.

\section{REFERENCES}

[1] Hafiz Ahmed, Rosane Ushirobira, Denis Efimov, Damien Tran, and Jean-Charles Massabuau. Velocity estimation of valve movement in oysters for water quality surveillance. IFAC-PapersOnLine, 48(11):333-338, 2015.

[2] Marco Tulio Angulo and Jorge X. Velasco-Hernandez. Robust qualitative estimation of time-varying contact rates in uncertain epidemics. Epidemics, pages 98 - 104, 2018.

[3] Pierre-Alexandre Bliman and Bettina D'Avila Barros. Interval observers for SIR epidemic models subject to uncertain seasonality. In Filippo Cacace et al., editor, Positive Systems, volume 471 of Lecture Notes in Control and Information Sciences. Springer, 2017.

[4] François Boulier. Étude et implantation de quelques algorithmes en algèbre différentielle. $\mathrm{PhD}$ thesis, 1994.

[5] François Boulier. Differential Elimination and Biological Modelling. Radon Series on Computational and Applied Mathematics (Gröbner Bases in Symbolic Analysis), 2:111-139, October 2007.

[6] François Boulier, Daniel Lazard, François Ollivier, and Michel Petitot. Computing representations for radicals of finitely generated differential ideals. Applicable Algebra in Engineering, Communication and Computing, 20(1):73, Mar 2009.

[7] François Boulier, François Lemaire, Markus Rosenkranz, Rosane Ushirobira, and Nathalie Verdière. On Symbolic Approaches to Integro-Differential Equations. In Niculescu Silviu-Iulian and Ditzinger Thomas, editors, Algebraic and Symbolic Computation Methods in Dynamical Systems, Advances in Delays and Dynamics. Springer, 2017.

[8] S Diop, M Fliess, et al. On nonlinear observability. In Proc. 1st Europ. Control Conf, pages 152-157, 1991.

[9] Torkel Glad and Lennart Ljung. Parametrization of nonlinear model structures as linear regressions. IFAC Proceedings Volumes, 23(8, Part 3):317 - 321, 1990. 11th IFAC World Congress on Automatic Control, Tallinn, 1990 - Volume 3, Tallinn, Finland.

[10] Evelyne Hubert. Algebra and algorithm for singularities of implicit differential equations. $\mathrm{PhD}$ thesis, Institut National Polytechnique Grenoble, 41997.

[11] Matt J. Keeling and Pejman Rohani. Modeling Infectious Diseases in Humans and Animals. Princeton University Press, 2008.

[12] Maria S. Aronna, Pierre-Alexandre Bliman. Interval observer for uncertain time-varying SIR-SI epidemiological model of vector-borne disease. In proceedings of ECC'18, the 16th annual European Control Conference, June 2018.

[13] François Ollivier. Some theoretical problems in effective differential algebra and their relation to control theory. In Proc. Nolcos, 1992.

[14] Wilfrid Perruquetti, Thierry Floquet, and Emmanuel Moulay. Finitetime observers: Application to secure communication. IEEE Trans. Automatic Control, 53(1):356-360, 2008.

[15] Pierre-Alexandre Bliman, Denis Efimov and Rosane Ushirobira. A class of nonlinear adaptive observers for SIR epidemic model. In proceedings of ECC'18, the 16th annual European Control Conference, June 2018. 
[16] Gianluigi Pillonetto and Maria Pia Saccomani. Input estimation in nonlinear dynamical systems using differential algebra techniques. Automatica, 42(12):2117 - 2129, 2006.

[17] Joseph F. Ritt. Differential Algebra. American Mathematical Society: Colloquium publications. American Mathematical Society, 1950.

[18] Maria Pia Saccomani, Stefania Audoly, and Leontina D'Angiò. Parameter identifiability of nonlinear systems: the role of initial conditions. Automatica, 39(4):619 - 632, 2003.

[19] Rosane Ushirobira, Denis Efimov, Géry Casiez, Nicolas Roussel, and Wilfrid Perruquetti. A forecasting algorithm for latency compensation in indirect interaction. In In proceedings of ECC'16, the 15th annual European Control Conference, page 6, 2016. 